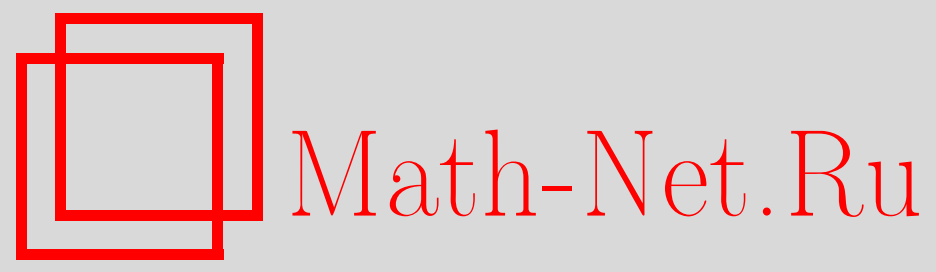

Е. В. Дубовова, Исследование процесса релаксации остаточных напряжений в поверхностно упрочнённом слое кругового отверстия пластины при виброползучести, Вестн. Сам. гос. техн. ун-та. Сер. Физ.-мат. науки, 2012, выпуск 2(), 78-84

DOI: https://doi.org/10.14498/vsgtu1093

Использование Общероссийского математического портала Math-Net.Ru подразумевает, что вы прочитали и согласны с пользовательским соглашением

http://www . mathnet.ru/rus/agreement

Параметры загрузки:

IP : 54.80 .73 .141

26 апреля 2023 г., 18:04:03

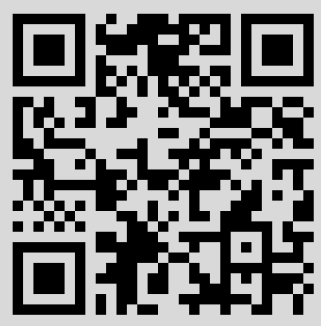




\title{
ИССЛЕДОВАНИЕ ПРОЦЕССА РЕЛАКСАЦИИ ОСТАТОЧНЫХ НАПРЯЖЕНИЙ В ПОВЕРХНОСТНО УПРОЧНЁННОМ СЛОЕ КРУГОВОГО ОТВЕРСТИЯ ПЛАСТИНЫ ПРИ ВИБРОПОЛЗУЧЕСТИ
}

\author{
E. В. Дубовова \\ Самарский государственный технический университет, \\ 443100, Россия, Самара, ул. Молодогвардейская, 244. \\ E-mail: elenad_86@inbox.ru
}

\begin{abstract}
Разработан и реализован метод расчёта релаксации остаточных напряжений в поверхностно упрочнённом слое кругового отверстия пластины в условиях ползучести при комбинированном действии статических и ииклических нагрузок. На основании идей декомпозиции и агрегирования расчёт релаксации остаточных напряжений в поверхностном слое кониентратора напряжений сводится $к$ решению трёх задач и склейки их решений. В процессе решения первой задачи определяется трёхмерное распределение полей остаточных напряжений и пластических дебормаций в упрочнённом слое кругового концентратора напряжений пластины. В процессе решения второй задачи определяется напряжённо-дебормированное состояние пластины с круговым отверстием при ползучести без учёта поверхностного упрочнённого слоя. При решении третъей задачи исследуется релаксация остаточных напряжений в поверхностно упрочнённом слое концентратора напряжений, дебормирующемся в режиме «жёсткого» нагружения при заданных значениях компонент тензоров дебормаций, которые определяются из решения второй задачи. Выполнен численный анализ влияния величины амплитудного значения ииклической составляющей на процесс релаксаиии остаточных напряжений, который позволяет сделать однозначный вывод об увеличении скорости релаксации остаточных напряжений в зависимости от ииклической составляющей.
\end{abstract}

Ключевые слова: остаточные напряжения, релаксация остаточных напряжений, кониентратор напряжений, пластина с круговым отверстием, виброползучесть.

Отверстия различной формы, используемые для сопряжения и крепления различных деталей, являются зонами повышенного внимания и для продления ресурса изделия подвергаются процедуре упрочнения. Сжимающие остаточные напряжения, наведённые методами поверхностного пластического деформирования, играют положительную роль при эксплуатации таких конструкций: при нормальной температуре они сохраняют свой уровень в течение длительного времени. Однако при повышенных температурах вследствие ползучести и различных термофлуктуационных процессов происходит релаксация (уменьшение по модулю) остаточных напряжений.

Реальные условия работы деталей машин сопровождаются вибрационным фоном, который существенно влияет на скорость накопления деформаций ползучести, долговечность конструкции и кинетику остаточных напряжений в условиях высокотемпературной ползучести. Таким образом, учёт вибрационной составляющей, наложенной на статическую нагрузку, при прогнозировании долговечности упрочнённых элементов конструкций в условиях высоких температур имеет определённый научный и практический интерес.

Елена Валеръяновна Дубовова, аспирант, каф. прикладной математики и информатики. 
Сформулируем цель настоящей работы: разработка метода оценки кинетики остаточных напряжений в условиях ползучести и виброползучести с учётом разрушения материала и накопления повреждённости в круговом концентраторе напряжения пластины (плиты).

Метод оценки кинетики остаточных напряжений основан на методе декомпозиции на тонкий упрочнённый слой и «тело» пластины и склейки решений нелинейных краевых задач [1]. Он сводится к решению следующих задач:

1) определение полей остаточных напряжений и пластических деформаций в концентраторах напряжений непосредственно после процедуры поверхностного пластического деформирования;

2) решение задачи неупругого деформирования (определение деформаций ползучести) пластины при приложении внешней нагрузки с учётом циклической составляющей без упрочнённого слоя;

3) расчёт кинетики остаточных напряжений в поверхностно упрочнённом слое концентратора пластины в режиме «жёсткого» нагружения (при заданных значениях компонент тензора деформаций на поверхности концентратора напряжений пластины, которые определяются из решения второй задачи).

Предложенная методика в настоящей работе апробирована на круговом концентраторе напряжений прямоугольной пластины. В качестве модельного материала пластины рассматривался сплав ЭИ698 при температуре $700{ }^{\circ} \mathrm{C}$.

Исходными данными для расчёта кинетики остаточных напряжений в упрочнённом слое концентратора напряжений пластин является распределение полей остаточных напряжений и пластических деформаций, образующихся в этом слое после процедуры упрочнения. Методика решения этой задачи подробно изложена в работе [2]. Согласно этой методике трёхмерное распределение полей остаточных напряжений и пластических деформаций в упрочнённом слое кругового концентратора напряжений пластины можно определить по одной (или двум) экспериментально измеренной компоненте остаточных напряжений.

Решение второй задачи производится методом конечных элементов $[3,4]$. В силу симметрии задачи рассматривалась четверть пластины (см. рис. 1). Геометрические размеры пластины $-80 \times 50 \times 5$ мм, радиус концентратора напряжений $-R=8$ мм. При

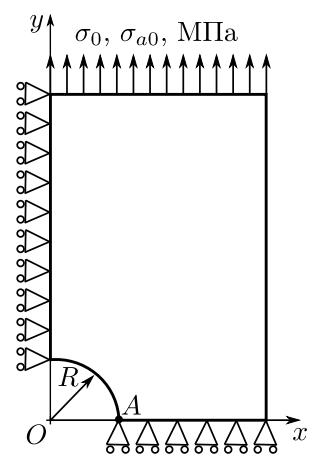
решении использовалась глобальная декартова система координат $O x y z$ с началом в центре пластины. Оси $O x, O y$ располагались в плоскости пластины, ось $O z$ - перпендикулярно. Внешняя распределённая нагрузка с квазистатической $\sigma_{0}$ и циклической $\sigma_{a 0}$ составляющими действовала в направлении оси $O y$ (см. рис. 1).

Для расчёта неупругой деформации в работе используется апробированная [5,6] модель виброползучести, конечно-элементный вариант которой для $n$-ного конечного элемента записывается так:

$$
\left\{\varepsilon^{(n)}\right\}=\left\{e_{a}^{(n)}\right\}+\left\{p^{(n)}\right\}
$$

Рис. 1. Схема закрепления и приложения нагрузки для четверти пластины с круговым концентратором напряжений 


$$
\begin{aligned}
& \left\{p^{(n)}\right\}=\left\{u^{(n)}\right\}+\left\{v^{(n)}\right\}+\left\{w^{(n)}\right\} ; \\
& \left\{\varepsilon^{(n)}\right\}=\left\{\varepsilon_{x}, \varepsilon_{y}, \varepsilon_{x y}\right\}^{\top},\left\{p^{(n)}\right\}=\left\{p_{x}, p_{y}, p_{x y}\right\}^{\top},\left\{u^{(n)}\right\}=\left\{u_{x}, u_{y}, u_{x y}\right\}^{\top}, \\
& \left\{v^{(n)}\right\}=\left\{v_{x}, v_{y}, v_{x y}\right\}^{\top},\left\{w^{(n)}\right\}=\left\{w_{x}, w_{y}, w_{x y}\right\}^{\top},\left\{e_{a}^{(n)}\right\}=\left\{e_{a x}, e_{a y}, e_{a x y}\right\}^{\top} ; \\
& e_{a i j}^{(n)}=\frac{1+\nu}{E} \sigma_{i j a}-\frac{\nu}{E} \sigma_{k k a} \delta_{k k} ; \\
& \left\{u^{(n)}\left(t_{s+1}\right)\right\}=\sum_{k}\left\{u_{k}^{(n)}\left(t_{s}\right)\right\}+\sum_{k}\left\{\Delta u_{k}^{(n)}\left(t_{s}\right)\right\} \text {, } \\
& \left\{\Delta u_{k}^{(n)}\left(t_{s}\right)\right\}=\lambda_{k}\left(a_{k} S^{n_{2}-1}\left[N_{\mu_{k}^{\prime}}\right]\left\{\sigma^{(n)}\right\}^{\top}-\left\{u_{k}^{(n)}\left(t_{s}\right)\right\}\right) \Delta t_{s} ; \\
& \left\{v^{(n)}\left(t_{s}\right)\right\}=\left[L^{(n)}\right]\left[M_{\mu_{k}^{\prime \prime}}\right]\left\{\beta^{(n)}\left(t_{s}\right)\right\}^{\top}, \quad\left\{\beta^{(n)}\left(t_{s}\right)\right\}=\sum_{k}\left\{\beta_{k}^{(n)}\left(t_{s}\right)\right\}, \\
& \left\{\beta_{k}^{(n)}\left(t_{s+1}\right)\right\}=\left\{\beta_{k}^{(n)}\left(t_{s}\right)\right\}+\left\{\Delta \beta_{k}^{(n)}\left(t_{s}\right)\right\} \\
& \left\{\Delta \beta_{k}\left(t_{s}\right)\right\}=\left\{\begin{array}{cl}
\lambda_{k}\left\{\Gamma\left(t_{s}\right)\right\} \Delta t_{s}, & \left\{\Gamma\left(t_{s}\right)\right\}\left\{\bar{\sigma}^{(n)}\right\}^{\top}>0, \\
0, & \left\{\Gamma\left(t_{s}\right)\right\}\left\{\bar{\sigma}^{(n)}\right\}^{\top} \leqslant 0,
\end{array}\right. \\
& \left\{\Gamma\left(t_{s}\right)\right\}=\left(b_{k} / \sigma^{*}\right)\left(S / \sigma^{*}\right)^{n_{2}-1}\left\{\bar{\sigma}^{(n)}\right\}-\left\{\beta_{k}^{(n)}\left(t_{s}\right)\right\} ; \\
& \left\{w^{(n)}\left(t_{s+1}\right)\right\}=\left\{w^{(n)}\left(t_{s}\right)\right\}+\left\{\Delta w^{(n)}\left(t_{s}\right)\right\} \text {, } \\
& \left\{\Delta w^{(n)}\left(t_{s}\right)\right\}=\left(c / \sigma^{*}\right)\left(S / \sigma^{*}\right)^{m_{1}-1}\left[N_{1 / 2}\right]\left\{\sigma^{(n)}\right\}^{\top} \Delta t_{s} ; \\
& \left\{\sigma^{(n)}\right\}=\left\{\sigma_{0}^{(n)}\left(t_{s}\right)\right\}\left(1+\omega\left(t_{s}\right)\right) ; \quad\left\{\sigma_{a}^{(n)}\right\}=\left\{\sigma_{a 0}^{(n)}\left(t_{s}\right)\right\}\left(1+\omega\left(t_{s}\right)\right) ; \\
& \Delta \omega\left(t_{s}\right)=\alpha\left(S_{0}\right)\left\{\sigma^{(n)}\right\}\left\{\Delta p^{(n)}\left(t_{s}\right)\right\}^{\top}+0,5 g\left(S_{0}, S_{a 0}, f\right)\left\{\sigma_{a}^{(n)}\right\}\left\{e_{a}^{(n)}\right\}^{\top} f \Delta t .
\end{aligned}
$$

Здесь $\left\{u^{(n)}\right\},\left\{v^{(n)}\right\},\left\{w^{(n)}\right\}$ - вязкоупругая, вязкопластическая и вязкая составляющие вектора деформаций виброползучести $\left\{p^{(n)}\right\}$ для $n$-ного конечного элемента; $\left\{\sigma^{(n)}\right\},\left\{\sigma_{0}(n)\right\}$ - векторы истинной и номинальной квазистатической составляющей тензора напряжений; $\left\{\sigma_{a}^{(n)}\right\}=\left\{\sigma_{x}, \sigma_{y}, \sigma_{x y}\right\}^{\top},\left\{\sigma_{a 0}^{(n)}\right\}-$ амплитудные значения векторов истинной и номинальной циклической составляющей тензора напряжений; $S, S_{0}$ - интенсивности истинных и номинальных квазистатических напряжений; $S_{a}, S_{a 0}$ - интенсивности истинных и номинальных циклических напряжений; $\lambda_{k}, a_{k}, b_{k}, c, n_{2}, m_{1}, \sigma^{*}$ - реологические константы материала, при помощи которых описываются первая и вторая стадии ползучести и обратимая часть деформации ползучести; $\omega(t)-$ скалярный параметр повреждённости материала; $\alpha\left(S_{0}\right), g\left(S_{0}, S_{a 0}, f\right)$ - параметры материала, контролирующие процессы разупрочнения (описывающие третью стадию в условиях виброползучести); $E$ - модуль упругости; $\nu$ - коэффициент Пуассона; $f$ - частота изменения $\sigma_{a} ;\left\{\bar{\sigma}^{(n)}\right\}=\left\{\sigma_{1}, \sigma_{2}\right\}^{\top}$ - вектор главных напряжений внутри конечного элемента; $\left\{\beta^{(n)}\right\}=\left\{\beta_{1}, \beta_{2}\right\}^{\top}$ - вектор активных вязкопластических деформаций элемента в главных осях. В вышеприведённых соотношениях используются следующие матрицы:

$$
\begin{gathered}
{\left[N_{\kappa}\right]=\left[\begin{array}{ccc}
1 & -\kappa & 0 \\
-\kappa & 1 & 0 \\
0 & 0 & 1+\kappa
\end{array}\right], \quad\left[M_{\kappa}\right]=\left[\begin{array}{cc}
1 & -\kappa \\
-\kappa & 1
\end{array}\right],\left[L^{(n)}\right]=\frac{1}{k_{2}}\left[\begin{array}{cc}
1 & k_{1}^{2} \\
k_{1}^{2} & 1 \\
k_{1} & -k_{1}
\end{array}\right],} \\
k_{1}=\left(\sigma_{1}-\sigma_{x}\right) / \sigma_{x y}, \quad k_{2}=1+k_{1}^{2} .
\end{gathered}
$$


Для прогнозирования времени разрушения $t_{*}$ конечного элемента используется критерий разрушения энергетического типа:

$$
\int_{0}^{t_{*}} \frac{\left\{\sigma^{(n)}\right\} d\left\{p^{(n)}\right\}^{\top}}{A_{*}^{c}\left(S_{0}\right)}+\frac{1}{2} \int_{0}^{t_{*}} \frac{\left\{\sigma_{a}^{(n)}\right\}\left\{e_{a}^{(n)}\right\}^{\top} f \Delta t}{A_{*}^{y}\left(S_{0}\right)}=1,
$$

где $A_{*}^{c}\left(S_{0}\right), A_{*}^{y}\left(S_{0}\right)$ - критические величины работ напряжений в условиях стационарной ползучести и виброползучести.

Задача ползучести решается методом начальных деформаций [4]. Методика определения реологических констант и параметров материала, а также их значения для сплава ЭИ 698 при $T=700^{\circ} \mathrm{C}$ приведены в работах $[6,7]$.

Для проверки методики конечно-элементного расчёта рассматривалось одноосное растяжение пластины без концентратора. При этом кривые деформирования пластины при её одноосном растяжении, полученные методом конечных элементов, практически полностью совпадали с кривыми, полученными при одноосных испытаниях образцов [6].

Для решения третьей задачи использовались следующие гипотезы:

1) поскольку компоненты тензора остаточных напряжений за пределами тонкого поверхностного слоя концентратора малы, деформирование пластины внешней растягивающей силой происходит в целом так же, как если бы упрочненного слоя не было;

2) в процессе упрочнения вторичные пластические деформации в области сжатия не возникают, а наведённые пластические деформации не оказывают влияния на процесс развития деформаций ползучести.

Как и в случае с цилиндрическим образцом [8], в локальной цилиндрической системе координат, связанной с центром кругового концентратора напряжений, для деформаций поверхностного слоя можно записать

$$
\varepsilon_{i}(t)+\varepsilon_{i}^{0}(r)=q_{i}(r)+e_{i}^{\mathrm{res}}(r, t)+p_{i}^{\mathrm{res}}(r, t) \quad i=r, \theta, z,
$$

где $e_{i}^{\mathrm{res}}(r, t), p_{i}^{\mathrm{res}}(r, t)$ - компоненты тензоров упругих деформаций, деформаций ползучести (виброползучести), которые рассчитываются через напряжения $\sigma_{i}^{\text {res }}(r, t)$ в поверхностном слое; $q_{i}(r)$ - компоненты остаточных пластических деформаций, определяемые в результате решения первой задачи (см. [2]). Здесь величины $\varepsilon_{i}^{0}(r)$ - компоненты тензора полных деформаций, наведённых в поверхностном слое после процедуры поверхностного пластического деформирования. При этом принимается гипотеза плоских сечений: $\varepsilon_{z}^{0}(r)=e_{z}^{0}(r)+q_{z}(r)=\varepsilon_{z}^{0}=$ const.

В соотношениях (1) кинетика величин $\varepsilon_{i}(t)$ определяется из решения второй задачи. Так как приложенная к пластине нагрузка действует лишь в её плоскости, на поверхности концентратора напряжений при $r=R$ реализуется одноосное напряжённое состояние, при этом величина $\varepsilon_{\theta}(t)$ в каждой конкретной точке концентратора напряжений определяется из решения краевой задачи, а величины $\varepsilon_{z}(t), \varepsilon_{\theta}(t)$ обусловлены лишь пуассоновским сужением материала и будут определяться так:

$$
\varepsilon_{i}(t)=-\mu e_{\theta}-\mu^{\prime} u_{\theta}(t)-\mu^{\prime \prime} v_{\theta}(t)-0,5 w_{\theta}(t), \quad i=r, z .
$$

Здесь $\mu^{\prime}=0,38$ и $\mu^{\prime \prime}=0,42$ - коэффициенты Пуассона для обратимой $u$ и необратимой $v$ компонент деформации ползучести соответственно. 
Выражая $e_{z}^{\mathrm{res}}(r, t), e_{\theta}^{\mathrm{res}}(r, t), e_{r}^{\mathrm{res}}(r, t)$ из (1) через $\sigma_{r}^{\mathrm{res}}(r, t), \sigma_{\theta}^{\mathrm{res}}(r, t), \sigma_{r}^{\mathrm{res}}(r, t)$ по закону Гука (аналогично случаю цилиндрического образца [8]), получим систему для определения кинетики остаточных напряжений $\sigma_{i}^{\mathrm{res}}(r, t)$ :

$$
\sigma_{i}^{\mathrm{res}}(r, t)=\frac{A_{i}(r, t)}{1+\mu}+\mu \frac{A_{r}(r, t)+A_{z}(r, t)+A_{\theta}(r, t)}{(1+\mu)(1-2 \mu)}, \quad i=r, z, \theta,
$$

где $A_{i}(r, t)=E\left(\varepsilon_{i}(t)+\varepsilon_{i}^{0}(r)-q_{i}(r)-p_{i}^{r}(r, t)\right)$.

На рис. 2-5 приведены результаты расчёта (временные срезы распределения $\sigma_{\theta}^{\text {res }}$ и $\sigma_{z}^{\text {res }}$ по глубине упрочнённого слоя $h$ при различных значениях амплитудной величины $\sigma_{a 0}$ циклической составляющей нагрузки) процесса релаксации для рассматриваемой пластины в точке $A$ (см. рис. 1) поверхности кругового концентратора напряжений при растягивающей квазистационарной нагрузке $\sigma_{0}=140 \mathrm{MПа} \mathrm{и} \mathrm{различных} \mathrm{амплитудных} \mathrm{значениях}$ циклической составляющей $\sigma_{a 0}$ при $T=700^{\circ} \mathrm{C}$.

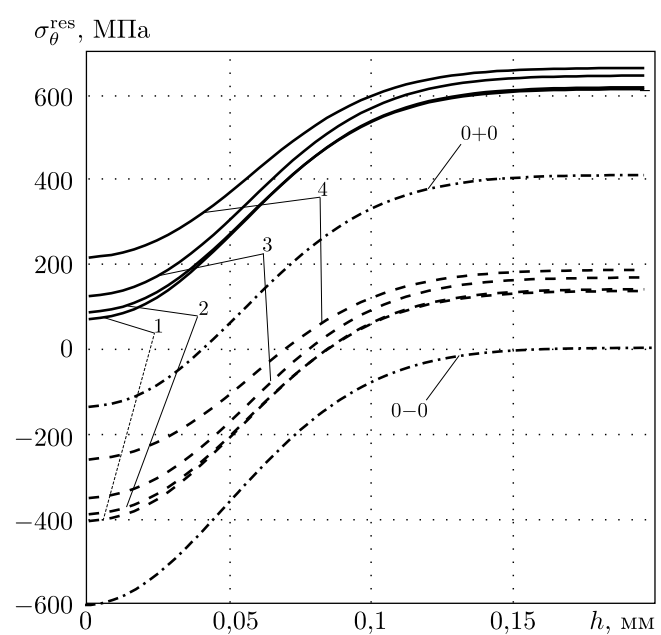

Рис. 2. Распределение $\sigma_{\theta}^{\text {res }}$ в момент времени $t=70$ ч (сплошные линии) и после разгрузки $t=70-0$ ч (штриховые линии) для точки $A$ : 1) $\sigma_{a 0}=0$; 2) $\sigma_{a 0}=25 \mathrm{MПа;}$ 3) $\sigma_{a 0}=50 \mathrm{M \Pi а}$;) $\sigma_{a 0}=75 \mathrm{M \Pi а}$

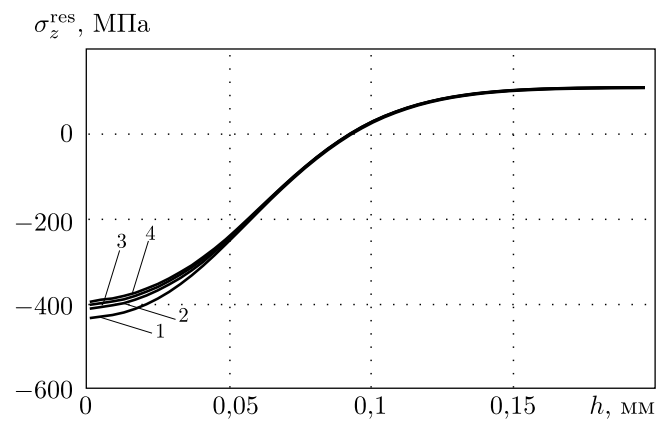

Рис. 4. Распределение $\sigma_{z}^{\text {res }}$ в момент времени $t=70$ ч для точки $A:$ 1) $\sigma_{a 0}=0$;2) $\sigma_{a 0}=$ $=25 \mathrm{M \Pi а}$; 3) $\sigma_{a 0}=50 \mathrm{M \Pi а}$; 4) $\sigma_{a 0}=75 \mathrm{M \Pi а}$

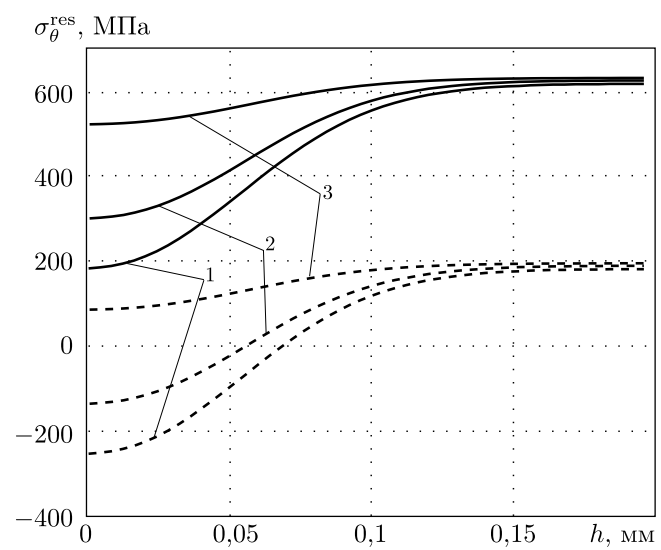

Рис. 3. Распределение $\sigma_{\theta}^{\text {res }}$ в момент времени $t=100$ ч (сплошные линии) и после разгрузки $t=100-0$ ч (штриховые линии) для точки $A$ : 1) $\sigma_{a 0}=0$; 2) $\sigma_{a 0}=25 \mathrm{MПа;}$ 3) $\sigma_{a 0}=50 \mathrm{M \Pi а}$

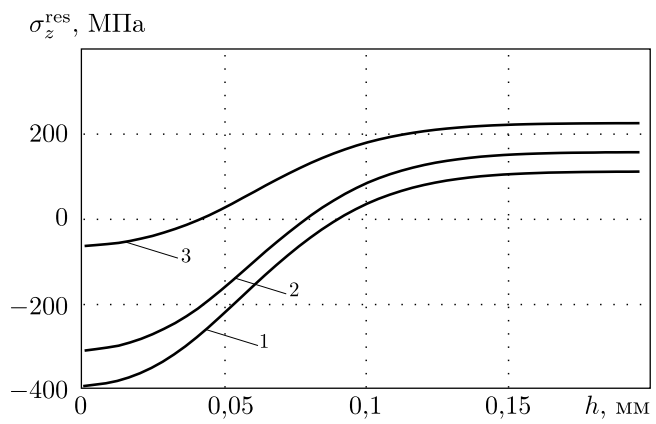

Рис. 5. Распределение $\sigma_{z}^{\text {res }}$ в момент времени $t=100$ ч для точки $A: 1) \sigma_{a 0}=0$; 2) $\sigma_{a 0}=25 \mathrm{M \Pi а}$; 3) $\sigma_{a 0}=50 \mathrm{M \Pi а}$ 
На рис. 2 приведены расчётные распределения компоненты $\sigma_{\theta}^{\text {res }}$ через 70 ч после приложения нагрузки (сплошные линии) и после разгрузки - снятия внешней нагрузки (штриховые линии). После разгрузки компонента $\sigma_{\theta}^{\text {res }}$ остаётся сжимающей в области, прилегающей к поверхности концентратора напряжений, для всех рассмотренных значений амплитудной величины $\sigma_{a 0}$ циклической составляющей нагрузки. Кроме этого, на рис. 2 приведены распределение компоненты $\sigma_{\theta}^{\text {res }}$ непосредственно после процедуры упрочнения (при $t=0-0$, линия $0-0)$ и распределение в момент нагружения (при $t=0+0$, линия $0+0)$. Как видно (штрих-пунктирные линии на рис. 2 ), в момент приложения нагрузки наблюдается упругий скачок.

Аналогичные кривые распределения компоненты $\sigma_{\theta}^{\text {res }}$ в момент времени $t=100$ ч приведены на рис. 3. Здесь видно, что после нагрузки с циклической составляющей $\sigma_{a 0}=50$ МПа остаточные напряжения после разгрузки в области, прилегающей к поверхности концентратора напряжений, становятся растягивающими. Отметим, что при нагрузке с циклической составляющей $\sigma_{a 0}=75$ МПа к этому времени рассматриваемая пластина согласно расчётам (решению второй задачи) уже разрушена.

Аналогичный характер влияния амплитудной величины циклической составляющей на релаксацию остаточных напряжений наблюдается и для компоненты $\sigma_{z}^{\text {res }}$ (см. рис. 4,5$)$, однако у этой компоненты отсутствует скачок в момент приложения нагрузки. На этих рисунках также наблюдается расслоение кривых в зависимости от величины циклической составляющей приложенной нагрузки. Компонента $\sigma_{z}^{\text {res }}$ остаётся сжимающей в области, прилегающей к поверхности концентратора напряжений, для всех рассмотренных значений циклической составляющей $\sigma_{a 0}$ вплоть до разрушения пластины.

Полученные расчётные данные позволяют сделать вывод, что увеличение амплитудной величины $\sigma_{a 0}$ циклической составляющей при одном и том же значении статической составляющей $\sigma_{0}$ приложенной нагрузки увеличивает скорость релаксации остаточных напряжений в концентраторе напряжений пластины, а также приводит к её преждевременному разрушению.

\section{БИБЛИОГРАФИЧЕСКИЙ СПИСОК}

1. Радченко В.П., Саушкин М.Н. Ползучесть и релаксация остаточных напряжений в упрочнённых конструкциях. М.: Машиностроение-1, 2005. 226 с. [Radchenko V.P., Saushkin M. N. Creep and Relaxation of Residual Stresses in Hardened Structures. Moscow: Mashinostroenie-1, 2005. 226 pp.]

2. Дубовова E. В., Смыслов $B . Ю$ Расчёт полей остаточных напряжений и пластических деформаций в поверхностно упрочнённом слое кругового концентратора плиты с учётом организации процесса поверхностного пластического деформирования / В сб.: Труды седъмой Всероссийской научной конферениии с международным участиeм. Часть 1: Математические модели механики, прочности и надёжности элементов конструкций / Матем. моделирование и краев. задачи. Самара: СамГТУ, 2010. С. 130-133. [Dubovova E. V., Smyslov V. Yu. Calculation of residual stresses and plastic deformations fields in the surface hardened layer of circular concentrator in plate taking into account the organization of surface plastic deformation process / In: Proceedings of the Seventh AllRussian Scientific Conference with international participation. Part 1 / Matem. Mod. Kraev. Zadachi. Samara: SamGTU, 2010. Pp. 130-133].

3. Segerlind L. J. Applied Finite Element Analysis. New York: John Wiley \& Sons, 1984. 427 pp.

4. Zienkiewicz O.C. The Finite Element Method in Engineering Science. New York: McGrawHill, 1972. 432 pp.

5. Radchenko V.P., Kichaev E. K., Simonov A. V. Energetic variant of the model of rheological 
deformation and destruction of metals under a joint action of static and cyclic loads // J. Appl. Mech. Tech. Phys., 2000. Vol. 41, no. 3. Pp. 531-537.

6. Радченко В.П., Кичаев П.Е. Энергетическая концепция ползучести и виброползучести металлов. Самара: СамГТУ, 2011. 157 с. [Radchebko V.P., Kichaev P. E. Energetic conception of the creep and vibrocreep of metals. Samara: SamGTU, 2011. 157 pp.]

7. Саушкин М. Н., Дубовова Е. В. Идентификация параметров, контролирующих процессы разупрочнения материала в условиях ползучести и виброползучести / В сб.: Труды пятой Всероссийской научной конференции с международным участием (29-31 мая 2008 г.). Часть 1: Математические модели механики, прочности и надёжности элементов конструкций / Матем. моделирование и краев. задачи. Самара: СамГТУ, 2008. С. 266272. [Saushkin M. N., Dubovova E. V. Identification of the parameters that control the process of softening of the material in terms of creep and vibrocreep/In: Proceedings of the Fifth All-Russian Scientific Conference with international participation (29-31 May 2008). Part 1 / Matem. Mod. Kraev. Zadachi. Samara: SamGTU, 2008. Pp. 266-272].

8. Саушкин M.Н., Дубовова E.В. Метод решения краевой задачи релаксации остаточных напряжений в упрочненном слое цилиндрического образца при виброползучести // Вестн. Сам. гос. техн. ун-та. Сер. Физ.-мат. науки, 2010. №1(20). С. 111-120. [Saushkin M.N., Dubovova E. V. A method of solving boundary value problem of residual stresses relaxation in the hardened layer of cylindrical specimen during vibrocreep // Vestn. Samar. Gos. Tekhn. Univ. Ser. Fiz.-Mat. Nauki, 2010. no.1(20). Pp. 111-120].

Поступила в редакцию $12 / \mathrm{II} / 2012$;

в окончательном варианте $-31 / \mathrm{V} / 2012$.

MSC: 74A10,74C05

\section{AN INVESTIGATION OF RESIDUAL STRESS RELAXATION PROCESSES WHICH ARISES IN PLATE CIRCLE HOLE SURFACE LAYER IN VIBROCREEP CONDITIONS}

\section{E. V. Dubovova}

Samara State Technical University,

443100, Samara, Molodogvardejskaya st., 244

E-mail: elenad_86@inbox.ru

Development and implementation of calculation method of residual stress relaxation in the surface hardened layer of plate hole under vibrocreep conditions with combine static and cyclic loading are presented. Based on the ideas of decomposition and aggregation, the calculation of the kinetics of relaxation of residual stresses in the hole surface layer is reduced to gluing solutions of three boundary value problems. While solving the first boundary value problem the three-dimensional distribution of residual stress fields and plastic strains in hole hardened layer of plate is defined. Solving of the second boundary value problem defines the stress-strain state of a hole hardened layer of plate during vibrocreep without taking into account the surface hardened layer. For the third boundary value problem the relaxation of residual stresses in surface hardening layer, deformable in the "hard" loading at given values of the strain tensor components, which are determined by the second boundary value problem solving, is investigated. The numerical analysis of the cyclic component amplitude value influence on residual stress relaxation process is completed. The numerical analysis presents a residual stress relaxation rate increasing according to cyclic component value.

Key words: residual stress, residual stress relaxation, stress concentrator, plate with circular hole, vibrocreep.

Original article submitted $12 / \mathrm{II} / 2012$; revision submitted $31 / \mathrm{V} / 2012$.

Elena V. Dubovova, Postgraduate Student, Dept. of Applied Mathematics and Informatics. 